\title{
Bonnet Canal Surfaces
}

\section{Bonnet Kanal Yüzeyler}

\author{
Gülşah Aydın Şekerci ${ }^{*}{ }^{*}$, Muradiye Çimdiker ${ }^{2}$ 『
}

${ }^{1}$ Süleyman Demirel University, Isparta, TURKEY

2 Kırklareli University, Kırklareli, TURKEY

Sorumlu Yazar / Corresponding Author*: gulsahaydin@sdu.edu.tr

Geliş Tarihi / Received: 10.07.2018 DOI:10.21205/deufmd. 2019216119

Kabul Tarihi / Accepted: 23.11.2018_Araștırma Makalesi/Research Article

Atıfșekli/How to cite: AYDIN ȘEKERCi, G., ÇIMDIKER, M. (2019). Bonnet Canal Surfaces. DEUFMD, 21(61), 195-200.

\begin{abstract}
We know that Bonnet surfaces are the surfaces which can admit at least one non-trivial isometry that preserves the principal curvatures in the Euclidean three-dimensional space. In this study, firstly, we have examined the required conditions for the canal surfaces, which are called the special swept surfaces, to be Bonnet surfaces. After that, we have defined the Bonnet canal surfaces in the Euclidean three-dimensional space and have obtained some special results for the Bonnet canal surfaces. We have studied the Bonnet canal surfaces, which will be formed, when the curve generating the canal surface is a special curve. In addition, we have given an example of the Bonnet canal surface by considering the conditions.
\end{abstract}

Keywords: Canal Surfaces, Bonnet Surfaces, Isothermic Surfaces

Öz

Bonnet yüzeylerin, Öklidyen üç boyutlu uzayda asli eğrilikleri koruyan en az bir ihmal edilebilir izometriyi kabul edebilen yüzeyler olduğunu biliyoruz. Bu çalışmada, öncelikle, özel bir süpürme yüzeyi olarak adlandırılan kanal yüzeylerin Bonnet yüzey olması için gereken koşulları inceledik. Sonrasında, Öklidyen üç boyutlu uzayında, Bonnet kanal yüzeylerini tanımladık ve Bonnet kanal yüzeyler için bazı özel sonuçlar elde ettik. Kanal yüzeyi oluşturan eğri özel bir eğri olduğunda oluşacak olan Bonnet kanal yüzeyleri inceledik. Ek olarak, şartları göz önüne alarak Bonnet kanal yüzeyin örneğini verdik.

Anahtar Kelimeler: Kanal Yüzeyler, Bonnet Yüzeyler, İzotermik Yüzeyler

\section{Introduction}

Bonnet [1] proposed a problem which was about classification of non-trivial one parameter families of isometries preserving both principal curvatures. But, it was restated as mean curvature instead of both principal curvatures, since Gaussian curvature is preserved by isometries. Although Bonnet raised this problem, the term "Bonnet surface" is firstly used by Lalan [2] and he defined as "Bonnet surface is a surface which enables the isometric transformation that preserves the mean curvature". After that, many people $[1,3,4]$ have studied these surfaces. Also, this topic has been examined in Minkowsi space. One of its most recent examples was done by Ersoy and Eren [5]. They worked on timelike tangent developable surfaces to be timelike Bonnet surfaces.

According to the obtained results about the Bonnet surfaces, researchers have been 
DEU FMD 21(61), 195-200, 2019

categorised them into three types. The first of them is the surfaces with constant mean curvature where the plane and the sphere are excluded. If the surfaces are both the isothermic and the Weingarten surfaces with non-constant mean curvature, then these surfaces are isometric to surfaces of revolution. So, it is the second type. In this situation, they have infinite number of Bonnet nets. The last type is related to the surfaces with non-constant mean curvature which permit a single non-trivial isometry. If a surface is one of the three types mentioned above, then it is called a Bonnet surface [6].

The set of all surfaces, which are non-trivially isometric to each other by conservation of the principal curvatures, is called a B-family. Such two surfaces are said to be associated with each other. If an orthogonal net on the surface consists of the lines of curvature of an associated surface, then they are called B-nets or Bonnet nets $[1,3,4,6]$.

A surface occurs when we sweep a sphere on the space curve and that surface is called canal surface. It has been intensively studied by mathematicians as well as engineers since it is used in many fields, like the surface modeling for CAD/CAM, shape reconstruction [7]. One of these studies was done by Makeawa et.al. [8] They examined the regularity of pipe (tube) surfaces and for this, they have presented the required conditions. Then, Xu et.al. [9] studied on some algebraic, geometric properties of canal surfaces. Specially, they obtained simple expressions of the area and Gausian curvature for the canal surfaces, which are very useful results. Doğan and Yaylı gave the relations between parameter curves and lines of curvature on canal surfaces. Morever, they investigated tubular surfaces with Bishop frame [10-11].

In this study, firstly, we have examined the required conditions for canal surfaces, which are a special swept surface, to be Bonnet surfaces. Secondly, we have analyzed Bonnet canal surfaces in the Euclidean three-dimensional space and have obtained some special results for the Bonnet canal surfaces.

\section{Material and Method}

The canal surface is a surface which consists of cover of a family of the spheres. Specially, the centers of these spheres are located on a space curve. It is a surface of revolution when the centers of spheres is on the straight line.
A canal surface $X$ is parametrized by

$$
\begin{aligned}
X(u, v)=p(u)+ & r(u)\left[\sqrt{1-r^{\prime}(u)^{2}} \boldsymbol{n}(u) \cos v\right. \\
& +\sqrt{1-r^{\prime}(u)^{2}} \boldsymbol{b}(u) \sin v \\
& \left.-r^{\prime}(u) \boldsymbol{e}(u)\right]
\end{aligned}
$$

where $p(u)$ is the unit speed curve, $\{\boldsymbol{e}, \boldsymbol{n}, \boldsymbol{b}\}$ is Frenet frame of $p(u)$ and $r(u)$ is called radius function of $X$. Here, $\boldsymbol{e}=\boldsymbol{e}(u)$ is the tangent vector, $\boldsymbol{n}=\boldsymbol{n}(u)$ is the normal vector and $\boldsymbol{b}=$ $\boldsymbol{b}(u)$ is the binormal vector [7]. So, in [11], the coefficients of the first fundamental form for canal surfaces are written as

$$
\begin{aligned}
& E=\left[\begin{array}{c}
\kappa(u) r(u) \sqrt{1-r^{\prime 2}(u)} \cos v \\
+\left(r(u) r^{\prime}(u)\right)^{\prime}-1
\end{array}\right]^{2} \\
& +\left[\begin{array}{l}
r(u) \sqrt{1-r^{\prime 2}(u) \tau(u)} \\
+r(u) r^{\prime}(u) \kappa(u) \sin v
\end{array}\right]^{2} \\
& +\left[\begin{array}{c}
\left(r(u) \sqrt{1-{r^{\prime}}^{2}(u)}\right)^{\prime} \\
-r(u) r^{\prime}(u) \kappa(u) \cos v
\end{array}\right]^{2} \\
& F=\left[\begin{array}{c}
r^{2}(u)\left(1-{r^{\prime}}^{2}(u)\right) \tau(u)+ \\
r^{2}(u) r^{\prime}(u) \sqrt{1-r^{\prime 2}(u)} \kappa(u) \sin v
\end{array}\right], \\
& G=r^{2}(u)\left(1-r^{\prime 2}(u)\right) .
\end{aligned}
$$

Let $p(u)=(x(u), y(u))$ be a planar curve with arc length. So, its Frenet formulas have the following form

$\mathbf{p}^{\prime}(\mathrm{u})=\mathbf{e}(\mathrm{u})$,

$\mathbf{e}^{\prime}(\mathrm{u})=\kappa(\mathrm{u}) \mathbf{n}(\mathrm{u})$,

$\mathbf{n}^{\prime}(u)=-\kappa(u) \mathbf{e}(u)$.

When we assume $\boldsymbol{b}^{\prime}(u)=0, p(u)$ is a planar curve. If $r(u)$ is a constant and is given as $r$, then $X(u, v)$ is called a tube or pipe surface [10]. It is written as the following.

$$
\begin{aligned}
X(u, v)=p(u)+ & r[\boldsymbol{n}(u) \cos v \\
& +\boldsymbol{b}(u) \sin v],
\end{aligned}
$$

where $r$ is called the constant radius of the spheres [12]. 
DEU FMD 21(61), 195-200, 2019

Morever, the theorems and definitions, which will be used in the study of Bonnet surface, are given as follows.

Definition 2.1. If a surface in $\mathbb{R}^{3}$ has the coefficients of fundamental forms which satisfy the conditions $E=G, F=0,(M=0)$, then it is called isothermic [13].

Also, the isothermic surface provides the condition $\frac{\partial^{2}}{\partial u \partial v}\left(\log \frac{G}{E}\right)=0$ [14].

Theorem 2.2. The coordinate curves of a surface are line of curvature in a neighborhood of a nonumbilical point if and only if $F=M=0$, where $\mathrm{F}$ and $\mathrm{M}$ are the coefficients of the first and second fundamental form, respectively [12].

Definition 2.3. A net on a surface which satisfies the following conditions

$E=G, F=0, M=c \neq 0$

is called an A-net, where the coefficients of the fundamental forms are $E, G, F, M$ and $c$ is a constant [13].

Theorem 2.4. A surface is a Bonnet surface with no umbilic points if and only if the surface has an A-net [13].

According to this theorem, if the surface has an A-net, then it is a Bonnet surface. Using this theorem, a Bonnet surface is obtained that it permits a single non-trivial isometry. When the surface has more than one A-net, it is isothermic. If this surface is both isothermic and Weingarten surface, then it has infinite number of Bonnet nets, and so, it is Bonnet surface [6].

\section{Discussion and Conclusion}

In this section, we will obtain the Bonnet canal surface.

Definition 3.1. Canal surfaces that provide the properties of Bonnet surfaces, are called Bonnet canal surfaces.

To determine whether the canal surface is Bonnet surface, we work the isothermic Weingarten surfaces and the surfaces that permit a single non-trivial isometry for canal surfaces with non-constant mean curvature.

Firstly, let us obtain isothermic Weingarten surfaces for the canal surfaces. The coefficient $F$ for isothermic surface is zero. To be $F=0$ in (3), we have the following cases:

Case 1. If we assume $r^{\prime}=0$ and $\tau=0$, then $F=0$.
Case 2. If we assume $\kappa=0$ and $\tau=0$, then

$F=0$.

Case 3. If we assume $r^{\prime}=1$, then $F=0$.

Now, we will investigate for the above three cases of the canal surface to be a Bonnet surface.

For the case 1., let $r^{\prime}=0$ and $\tau=0$.

Since $r(u)$ is a constant, $X(u, v)$ is called a tube or pipe surface and it can be written as

$$
\begin{aligned}
X(u, v) & =p(u) \\
& +r\left[\begin{array}{c}
\boldsymbol{n}(u) \cos v \\
+\boldsymbol{b}(u) \sin v
\end{array}\right] .
\end{aligned}
$$

Morever, due to $\tau=0, p(u)$ is the planar curve. Using the equation (5), we have the first derivatives of the surface $X(u, v)$ as follows:

$$
\begin{aligned}
& \boldsymbol{X}_{\boldsymbol{u}}=\boldsymbol{e}(u)[1-r \kappa(u) \cos v], \\
& \boldsymbol{X}_{\boldsymbol{v}}=r[-\boldsymbol{n}(u) \sin v+\boldsymbol{b}(u) \cos v] .
\end{aligned}
$$

Using (8), then we have

$$
\begin{aligned}
& E=\left\langle\boldsymbol{X}_{\boldsymbol{u}}, \boldsymbol{X}_{\boldsymbol{u}}\right\rangle=(1-r \kappa(u) \cos v)^{2} \\
& G=\left\langle\boldsymbol{X}_{\boldsymbol{v}}, \boldsymbol{X}_{\boldsymbol{v}}\right\rangle=r^{2}
\end{aligned}
$$

Also, there exists the condition $\frac{\partial^{2}}{\partial u \partial v}\left(\log \frac{G}{E}\right)=0$ to be isothermic surface [14]. If we try to calculate, then we have

$$
\frac{\partial^{2}}{\partial u \partial v}\left(\log \frac{r^{2}}{(1-r \kappa(u) \cos v)^{2}}\right)=0
$$

This means that

$$
\frac{-2 r \kappa^{\prime}(u) \sin v}{(1-r \kappa(u) \cos v)^{2}}=0
$$

From (11), we obtain that $\kappa(u)$ is constant. Thus, the curvature of the planar curve is constant and so, the tube surface drawing by the planar curve is an isothermic surface.

The second derivates of the surface are

$$
\begin{aligned}
& \boldsymbol{X}_{\boldsymbol{u u}}=\boldsymbol{n}(u)\left(\kappa-r \kappa^{2} \cos v\right), \\
& \boldsymbol{X}_{\boldsymbol{u} v}=\boldsymbol{e}(u)(1+r \kappa \sin v), \\
& \boldsymbol{X}_{\boldsymbol{v} v}=-r[\boldsymbol{n}(u) \cos v+\boldsymbol{b}(u) \sin v] .
\end{aligned}
$$

For the canal surface, the surface normal vector $\boldsymbol{U}$ is given by 
DEU FMD 21(61), 195-200, 2019

$$
\begin{aligned}
\boldsymbol{U} & =\frac{X_{u} \times X_{v}}{\left\|X_{u} \times X_{v}\right\|} \\
& =-\boldsymbol{n}(u) \cos v-\boldsymbol{b}(u) \sin v .
\end{aligned}
$$

Using (12) and (13), the quantities of the second fundamental form are obtained as

$$
\begin{aligned}
L & =\left\langle\boldsymbol{X}_{\boldsymbol{u u}}, \boldsymbol{U}\right\rangle \\
& =\kappa \cos v(r \kappa \cos v-1), \\
M & =0
\end{aligned}
$$

and

$$
N=\left\langle\boldsymbol{X}_{v v}, \boldsymbol{U}\right\rangle=r .
$$

In that case, Gaussian curvature $K$ and mean curvature $H$ of the canal surface $X(u, v)$ are written by

$$
K=\frac{L N-M^{2}}{E G-F^{2}}=\frac{r \kappa \cos v}{r(1-r \kappa \cos v)}
$$

and

$$
\begin{aligned}
H & =\frac{N E-2 F M+G L}{2\left(E G-F^{2}\right)} \\
& =\frac{1-2 r \kappa \cos v}{r(1-r \kappa \cos v)}
\end{aligned}
$$

From (18), we see that $H$ is non-constant. Then, we need to show the necessary condition to be a Weingarten surface that is

$$
\frac{\partial K}{\partial u} \frac{\partial H}{\partial v}-\frac{\partial K}{\partial v} \frac{\partial H}{\partial u}=0
$$

When we consider the equations (17), (18) and (19), we find $\frac{\partial K}{\partial u}=0$ and $\frac{\partial H}{\partial u}=0$. Thus, the canal surfaces, which are isothermic and provide the condition $r^{\prime}=0$ and $\tau=0$, are Weingarten surfaces. Then, the following theorem is given.

Theorem 3.2. Let $p(u)$ be a planar curve, which generates tube or pipe surface. If the curvature of $p(u)$ is a constant, then the tube surface has infinite number of Bonnet nets. So, these surfaces have A-nets, i.e. they are Bonnet surfaces.

Thus, we write the following result since the planar curves with constant curvature are a circle or a straight line.
Corollary 3.3. Bonnet canal surfaces, which provide the conditions $r^{\prime}=0$ and $\tau=0$, are torus or right cylinder where $r$ is the radius function for canal surface, $\tau$ is the torsion of curve forming the canal surface.

So, after we make a suitable transformation for the Bonnet canal surface, it is modified to isothermal system of coordinates that is $E=G$. For this, if we replace $v \rightarrow f(v)$, the surface in equation (7) is written as

$$
\begin{aligned}
X(u, v) & =p(u) \\
& +r\left[\begin{array}{c}
\boldsymbol{n}(u) \cos f(v) \\
+\boldsymbol{b}(u) \sin f(v)
\end{array}\right] .
\end{aligned}
$$

According to the expression (20), the fundamental quantities of the surface become

$$
\begin{aligned}
E & =\left\langle\boldsymbol{X}_{\boldsymbol{u}}, \boldsymbol{X}_{\boldsymbol{u}}\right\rangle \\
& =(1-r \kappa \cos f(v))^{2} ;
\end{aligned}
$$

and

$$
G=\left\langle\boldsymbol{X}_{\boldsymbol{v}}, \boldsymbol{X}_{\boldsymbol{v}}\right\rangle=\left(r f^{\prime}(v)\right)^{2} .
$$

For $E=G$, we obtain the following equalities:

$$
1-r \kappa \cos f(v)=r f^{\prime}(v)
$$

or

$$
1-r \kappa \cos f(v)=-r f^{\prime}(v) .
$$

Let $1-r \kappa \cos f(v)=r f^{\prime}(v)$. Taking $f(v)=y$, the solution of the differential equation

$y^{\prime}=\frac{1-\kappa r \cos y}{r}$ is obtained as

$$
\begin{aligned}
& f(v) \\
& =2 \arctan \left(\sqrt{\frac{1-r \kappa}{1+r \kappa}} \tan \frac{\sqrt{1-(r \kappa)^{2}}}{2 r} v\right),
\end{aligned}
$$

Hence, the Bonnet canal surface could be parametrized as the following:

$$
\begin{aligned}
& X(u, v) \\
& =p(u) \\
& +r\left[\boldsymbol{n}(u) \cos \left\{2 \arctan \left(\sqrt{\frac{1-r \kappa}{1+r \kappa}} \tan \frac{\sqrt{1-(r \kappa)^{2}}}{2 r} v\right)\right\}\right. \\
& +\boldsymbol{b}(u) \sin \left\{2 \arctan \left(\sqrt{\frac{1-r \kappa}{1+r \kappa}} \tan \frac{\sqrt{1-(r \kappa)^{2}}}{2 r} v\right)\right\} .
\end{aligned}
$$


So, coefficients of the first fundamental form provide the condition $E=G, F=0$ for canal surface with the form (26). Here, $p(u)$ is the planar curve, radius function $r$ and curvature $\kappa$ are constants.

Special case: Let $1-r \kappa=1+r \kappa$. From here, we have $r=0$ or $\kappa=0$.

Since $r$ is not equal to zero, we get $\kappa=0$. So, if the surface is formed with a straight line, then we write the equation of the Bonnet canal surface as the following:

$$
\begin{aligned}
X(u, v) & =p(u) \\
& +r\left[\boldsymbol{n}(u) \cos \frac{v}{r}+\boldsymbol{b}(u) \sin \frac{v}{r}\right] .
\end{aligned}
$$

such that

$$
f(v)=2 \arctan \left(\tan \frac{v}{2 r}\right)=\frac{v}{r} .
$$

Then, the first derivates of the surface $X(u, v)$ are obtained

$$
\begin{aligned}
& \boldsymbol{X}_{\boldsymbol{u}}=\boldsymbol{e}(u) \\
& \boldsymbol{X}_{v}=-\boldsymbol{n}(u) \sin \frac{v}{r}+\boldsymbol{b}(u) \cos \frac{v}{r}
\end{aligned}
$$

So, we have $E=1, G=1$. The canal surface with constant radius, which is generated by straight, is the Bonnet canal surface. Actually, it is right cylinder.

Theorem 3.4. Let $X(u, v)$ be a regular tube surface formed by a planar curve. Then, we have the follows:

i) If the planar curve $p(u)$ of the Bonnet canal surface $X(u, v)$ is a geodesic, it is a cylinder.

ii)If the planar curve $p(u)$ of the Bonnet canal surface $X(u, v)$ is an asymptotic curve, then it is a cylinder.

Proof. i) Let $X(u, v)$ be a regular surface. The unit speed curve $p(u)$, which lies on $X(u, v)$, is a geodesic curve if and only if the acceleration vector $\boldsymbol{p}^{\prime \prime}(u)$ and the surface normal vector $\mathbf{U}$ are linearly dependent. In that case, let $p(u)$ be a geodesic curve on the tube. From the equations (5) and (13), we find

$$
\boldsymbol{U} \times \boldsymbol{p}^{\prime \prime}(u)=\kappa \sin v \mathbf{e}(\mathrm{u})=0
$$

According to the equation (30), we have $\kappa=0$ or $\sin v=0$.
If $\sin v=0$, then $X(u, v)$ could not be surface. Therefore, for the geodesic curve $p(u)$, if $\kappa=0$, then the regular tube is formed as a cylinder.

ii)Let $X(u, v)$ be a regular surface. The unit speed curve $p(u)$, which lies on $X(u, v)$, is an asymptotic curve if and only if the acceleration vector $\boldsymbol{p}^{\prime \prime}(u)$ is tangent to the surface. In that case, let the planar curve $p(u)$ be an asymptotic curve on the tube. From the equations (5) and (13), we have

$$
\left\langle\boldsymbol{U}, \boldsymbol{p}^{\prime \prime}(u)\right\rangle=-\kappa \cos v .
$$

From $\cos v \neq 0$, we take $\kappa=0$. In this case, we see that the regular tube becomes a cylinder.

For the case 2 ., let $\kappa=0$ and $\tau=0$. In that case, $p(u)$ is a straight line and these canal surfaces are surfaces of revolution.

The surfaces of revolution with non-constant mean curvature are the Bonnet canal surfaces since they are an isothermic Weingarten surfaces. So, we write the following result.

Corollary 3.5. The surfaces of revolution are Bonnet canal surfaces.

For the case 3., let $r^{\prime}=1$. We can take the linear radius function as $r(u)=u+c, c \in \mathbb{R}$. From here, the Bonnet canal surface is reparametrized as the following:

$$
X(u, v)=p(u)+u[-\boldsymbol{e}(u)]
$$

So, it is not a surface.

Example 3.6. Assume that the planar curve $p(u)=(\cos u, \sin u, 0)$ for the canal surface. We have

$$
\begin{aligned}
& \boldsymbol{p}^{\prime}(u)=(-\sin u, \cos u, 0)=\boldsymbol{e}(u), \\
& \boldsymbol{e}^{\prime}(u)=-(\cos u, \sin u, 0)=\boldsymbol{n}(u), \\
& \boldsymbol{n}^{\prime}(u)=-(-\sin u, \cos u, 0) .
\end{aligned}
$$

For $r=\frac{1}{5}$, the tube equation is given by

$$
X(u, v)=\left(\begin{array}{c}
\cos u\left(1-\frac{1}{5} \cos v\right), \\
\sin u\left(1-\frac{1}{5} \cos v\right), \\
\frac{1}{5} \sin v
\end{array}\right)
$$

Morever, canal surface, which is given by equation(34), is like in Figure 1. The 
parametrization (34) is the torus equation.

For this surface, the coefficients of the first fundamental form are obtained as

$$
E=\left(1-\frac{1}{5} \cos v\right)^{2}, G=\frac{1}{25}
$$

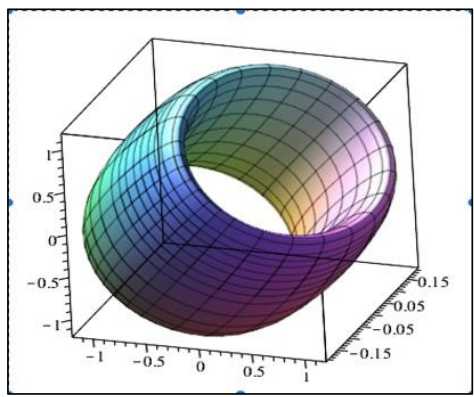

Figure 1. Example of the canal surface

For $E=G$, from (25), we have

$$
f(v)=2 \arctan \left(\sqrt{\frac{2}{3}} \tan \sqrt{6} v\right)
$$

Hence, the equation of the Bonnet canal surface is written by

$$
=\left(\begin{array}{c}
\cos u\left(1-\frac{1}{5} \cos \left(2 \arctan \left(\sqrt{\frac{2}{3}} \tan \sqrt{6} v\right)\right)\right), \\
\sin u\left(1-\frac{1}{5} \cos \left(2 \arctan \left(\sqrt{\frac{2}{3}} \tan \sqrt{6} v\right)\right)\right), \\
\frac{1}{5} \sin \left(2 \arctan \left(\sqrt{\frac{2}{3}} \tan \sqrt{6} v\right)\right) .
\end{array}\right)
$$

Morever, the Bonnet canal surface, which is given by equation (37) and is obtained by changing parametrization such that $E=G$, is in Figure 2.

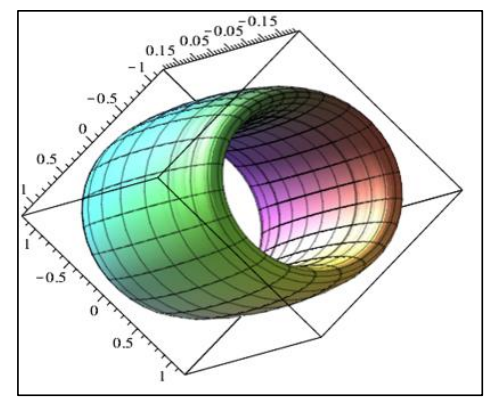

Figure 2. Example of Bonnet canal surface

So, we obtain the necessary conditions to be Bonnet surface of the canal surface with more than one A-net. Also, there is not an A-net from
$M=0$ for canal surfaces with $F=0$. Then, we have the following conclusion.

Corollary 3.7. There is not the canal surface with non-constant mean curvature that permit a single non-trivial isometry.

All the figures in this study is drawn by using Maple programme.

\section{References}

[1] Bonnet, 0., 1867. Memoire Sur la Theorie des Surfaces Applicables Sur une Surface Donee, Journal de l'Ecole Polytechnique, Paris, XLII, pp. 72-92.

[2] Lalan, V., 1949. Les Formes Minima des Surfaces d'ossian Bonnet, Bull. Soc. Math., France, Volume 77, pp. 102-127.

[3] Cartan, E., 1942. Sur la Couples de Surfaces Applicables Avec Conservation des Courbures Principales, Bulletin de la Societe Mathematique de France, Volume 66, pp. 55-85.

[4] Chern, S.S., 1985. Deformation of Surfaces Preserving Principal Curvatures, Differential Geometry and Complex Analysis, Berlin, pp. 155163. DOI:10.1007/978-3-642-69828-6_10.

[5] Ersoy, S., Eren, K., 2016. Timelike Tangent Developable Surfaces and Bonnet Surfaces, Abstract and Applied Analysis, Egypt, Volume 2016, pp. 1-7. DOI: $10.1155 / 2016 / 6837543$.

[6] Kanbay, F., 2005., Bonnet Ruled Surfaces, Acta Mathematica Sinica, English Series, Volume 21, No 3, pp. 623-630. DOI: 10.1007/s10114-004-0450-z.

[7] Ersoy, S., Eren, K., 2016. Timelike Tangent Developable Surfaces and Bonnet Surfaces, Abstract and Applied Analysis, Egypt, Volume 2016, pp. 1-7. DOI: $10.1155 / 2016 / 6837543$.

[8] Kim, Y.H., Liu, H., Qian, J., 2016. Some Characterizations of Canal Surfaces, Bulletin of the Korean Mathematical Society, Volume 53, No 2, pp. 461-477. DOI:10.4134/BKMS-2016-53-2-461.

[9] Maekawa, T., Patrikalakis, N.M., Sakkalis, T., Yu, G., 1998. Analysis and Applications of Pipe Surfaces, Computer Aided Geometric Design, Volume 15, pp. 437-458. DOI:10.1016/S0167-8396(97)-00042-3.

[10] Xu, Z., Feng, R., Sun, J.G., 2006. Analytic and Algebraic Properties of Canal Surfaces. Journal of Computational and Applied Mathematics, Volume 195, pp. 220-228. DOI: 10.1006/j.cam.2005.08.002.

[11] Dogan, F., Yayli, Y., 2011. On the Curvatures of Tubular Surface with Bishop Frame. Communications Faculty of Science, University of Ankara, Series A1, Volume 60, No 1, pp. 59-69.

[12] Dogan, F., Yayli, Y., 2017. The Relation Between Parameter Curves and Lines of Curvature on Canal Surfaces, Kuwait Journal of Science, Volume 44, No 1, pp. 29-35.

[13] do Carmo, P.M., 1976. Differential Geometry of Curves and Surfaces. Prentice-Hall, USA, 503p.

[14] Soyuçok, Z., 1995. The Problem of Non-Trivial Isometries of Surfaces Preserving Principal Curvatures, Journal of Geometry, Basel, Volume 52, pp. 173-188. DOI: 10.1007/BF01406838.

[15] Eisenhart, L.P., 1960. A Treatise On The Differential Geometry of Curves and Surfaces, Dover Publications, Inc. Newyork 347, pp. 229-231. 